\title{
La realidad de la brecha de conectividad en el ámbito educativo español: análisis de la situación actual
}

\author{
Connectivity divide reality in the Spanish educational area: \\ analysis of the current situation
}

RECIBIDO 2/2/2020 ACEPTADO 5/2/2020 PUBLICADO 1/6/2020

Roberto Soto Varela

Departamento de Lenguas Aplicadas y de Educación, Universidad de Nebrija, España

rsoto@nebrija.es

Mariano Sanz Prieto

Departamento de Pedagogía, Universidad Autónoma de Madrid, España

mariano.sanz@uam.es

Moussa Boumadan Hamed

Departamento de Pedagogía, Universidad Autónoma de Madrid, España

moussa.boumadan@uam.es

\section{RESUMEN}

El acceso a Internet se ha convertido en una herramienta indispensable en la sociedad en la que vivimos y, por consiguiente, las escuelas no han permanecido ajenas a este fenómeno. Su uso se ha convertido en una acción indispensable en el quehacer diario de una institución educativa.

Con este estudio se pretende analizar el estado actual de la conectividad del sistema educativo español y determinar la brecha de conectividad existente entre las diferentes comunidades de España.

Se ha podido observar que, desde una perspectiva general, a diferencia de las creencias del profesorado y alumnado de los distintos centros educativos, la conectividad goza de un estado de salud óptimo, con un porcentaje medio de disponibilidad de conexión en las aulas superior al 90\%. Sin embargo, no todas las comunidades autónomas gozan de la misma calidad de conexión, habiendo diferencias significativas dependiendo de la región en la que se ponga el foco. Esto supone un hándicap en el desarrollo de una secuencia didáctica mediada por tecnología en un aula.

PALABRAS CLAVE conectividad, internet, centros educativos.

\section{ABSTRACT}

The Internet has become an essential tool in the society in which we live, so schools have not been indifferent to this phenomenon. Its use has become an indispensable action in the daily work of an educational institution.

Through this study we aim to analyze the current state of connectivity in the Spanish education system, and to determine the connectivity divide that exists between the different Spanish regions.

It has been observed from a general perspective that, unlike teachers' and students' beliefs in the different educational centers, connectivity is in an optimal condition, with an average percentage of connection availability in the classrooms of 
more than 90\%. However, not all autonomous communities enjoy the same quality of connection, with significant differences depending on the region where the focus is placed. This is a disadvantage in developing a pedagogical sequence based on technology in the classroom.

KEYWORDS connectivity, internet, schools.

\section{INTRODUCCIÓN}

Las tecnologías de la información y comunicación (TIC) han adquirido un nivel de significación tan profundo que es cada más imprescindible su dominio para desenvolvernos en la sociedad en la que vivimos (Cabero et al., 2012).

En esta misma línea, queda patente que nunca se había dispuesto de tantas TIC a disposición como en la actualidad. Debido a esto, emergen con fuerza nuevos conceptos como transmedia que, según Orozco (2015), tiene que ver con el interés que tienen las audiencias de apropiarse y trasladar contenidos de unos espacios digitales a otros. En esta transición ocurre lo más interesante: son los individuos quienes trasladan lo que consideran oportuno y, además, deciden cómo hacerlo y en el proceso dotan a esos contenidos de nuevos significados. Tecnologías clásicas como la televisión y una más actual como la red social Twitter, son un claro ejemplo de este fenómeno, que desde un hashtag facilita la transición de contenidos de un canal a otro.

El crecimiento del sector tecnológico en la última década ha sido vertiginoso y, aunque las interacciones en redes sociales con amigos han generado un nicho de ocio muy agradable, fortaleciendo lazos con ellos, a la vez que intercambiando experiencias (Gómez García et al., 2015), el acoso en internet, la ciberintimidación, la incitación al odio... han surgido como serios problemas sociales. En consecuencia, parece que un amplio acceso a Internet no es suficiente para aprovechar de manera adecuada los beneficios que estos nuevos escenarios de interacción ofrecen (OCDE, 2019). Y es que uno de los mayores problemas a los que nos enfrentamos en esta nueva era de saturación tecnológica, es que estas tendencias de innovación no llegan a todos por igual. En este contexto, aunque no es en el único en el que se origina (Brown et al, 2020), surge una nueva marginalidad y exclusión social (Cabero Almenara y Ruiz Palmero, 2017).

Es precisamente en el nivel del acceso a la conectividad donde se ubica el núcleo central de este trabajo. Concretamente, indagar sobre el estado de la cuestión relacionada a la conectividad en territorio español será la incógnita a descifrar, teniendo en cuenta que, a lo largo del recorrido, se pueden desmentir varios mitos relacionados con la disponibilidad y calidad del acceso a la red de redes, llamada habitualmente Internet.

\section{REVISIÓN DE LA LITERATURA}

El programa One Laptop per Child (OLPC) es uno de los mayores aportes a la lucha contra la brecha digital. Fue creado hace más de una década por Nicholás Negroponte, director del Media Laboratory del Massachusetts Institute of Technology (MIT). Los resultados, después de una década de su implementación, no fueron los esperados. Solo Uruguay presenta resultados positivos, que normalmente son atribuidos al Plan Ceibal más que al OLPC. No obstante, el verdadero éxito del proyecto tiene más que ver con las lecciones aprendidas. Sus ideas inspiraron iniciativas que indiscutiblemente cambiaron la industria de la educación mediada por tecnología y plantaron algunas semillas que llevaron a investigar formas disruptivas de incorporar ar- 
tefactos tecnológicos al proceso de enseñanza y aprendizaje, cuestionando el paradigma tradicional de la mayoría de los sistemas educativos a nivel global (Ríos, 2018).

Probablemente, uno de los mayores inconvenientes de proyectos como OLPC y Plan Ceibal es la disponibilidad y calidad de la conexión a Internet. En este sentido, Facebook y The Economist Intelligence Unit (2019) elaboraron The Inclusive Internet Index 2019, un índice que recoge información sobre 100 países y proporciona un punto de referencia riguroso en relación con la inclusión de Internet a nivel nacional desde cuatro categorías: disponibilidad, asequibilidad, relevancia y capacidad. Cubre el $94 \%$ de la población mundial y su objetivo elemental es medir las percepciones sobre cómo el uso de Internet afecta a la vida de las personas.

Suecia lidera este ranking global, seguida de cerca por Singapur y Estados Unidos. Completan los primeros lugares Dinamarca, que se ubica en cuarta posición, y el Reino Unido en quinta. España ocupa el octavo lugar. Si se observa desde un nivel de continente, España se encuentra en quinta posición (figura 1), por detrás de Suecia, Dinamarca, Reino Unido y Finlandia, que desde la perspectiva global son el primero, cuarto, quinto y sexto.

Figura 1. España en The Inclusive Internet Index 2019. Tomada de Facebook y The Economist Intelligence Unit (2019)

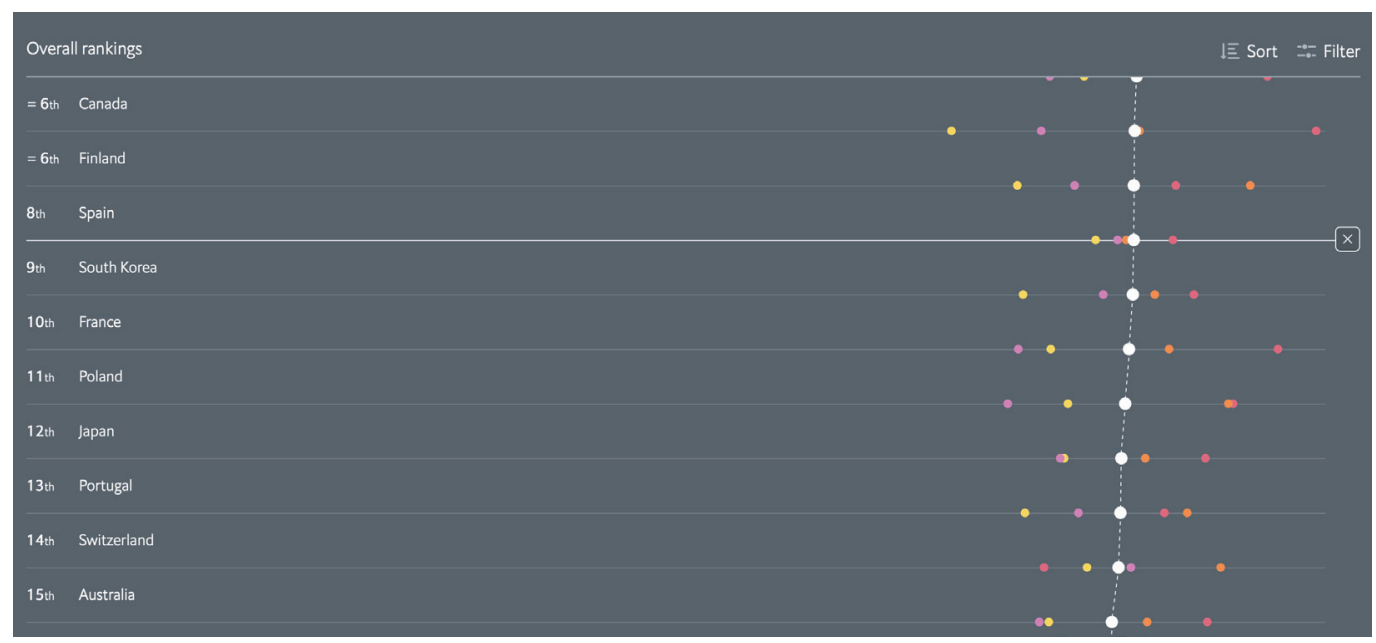

Situándonos en las variables de análisis, España destaca en cuanto a la disponibilidad, variable medida por la infraestructura disponible. Es cuarta teniendo por delante solo a Singapur, Suecia y Suiza. De acuerdo a la capacidad, variable medida por las habilidades para acceder a Internet y las políticas de apoyo, España también se sitúa en los primeros puestos; de hecho, es la primera empatada con Bélgica y Chile.

Sin embargo, España pierde muchos puestos en las otras dos variables. En cuanto a la asequibilidad, variable medida en términos de coste de acceso a Internet ocupa el puesto 20. En referencia a la relevancia, variable medida por la existencia de contenido relevante en el lenguaje local, ocupa el puesto 34. Estos datos se deben a la poca competitividad del mercado español en relación a la oferta de Internet y el poco posicionamiento de los contenidos generados en la lengua local.

Fundación Telefónica (2019), en su informe Sociedad Digital en España 2018, aporta una radiografía del estado de la disponibilidad de tecnología y conectividad en el panorama español. Destaca la aparición del $5 \mathrm{G}$ y su potencial para transformar y generar nuevos escenarios de uso de herramientas digitales. La inteligencia artificial, representada por los altavoces inteligentes, es otro de los grandes pilares destacados de este momento de desarrollo tecnológico. Además, se remarca también la necesidad de un marco ético de 
actuación debido a que muchas facetas de nuestras vidas se desarrollan en el plano digital, referencia a las ciudades inteligentes. Definitivamente hemos pasado del usuario digital al ciudadano digital.

Situándonos en el plano educativo, Alexander et al. (2019), en el Informe Horizon 2019, señalan a la equidad digital como uno de los desafíos importantes que impiden la adopción de tecnología en la educación superior. Con esta variable se refieren al acceso a la tecnología, particularmente a una conectividad de banda ancha suficiente para permitir acceder a un contenido imparcial y sin censura y, en consecuencia, facilitar a todos las plenas participaciones en la World Wide Web. El acceso a banda ancha sigue siendo globalmente desigual cuando la observamos desde variables como los ingresos, la educación, el género o la edad, entre otros.

En este mismo sentido, Unesco (2018) ha creado el proyecto de Definición de Indicadores de Universalidad de Internet. Un marco de indicadores mediante el cual evaluar los niveles de cumplimiento, en los diferentes países, de los cuatro principios fundamentales denominados DAAM (ROAM en inglés) incluidos en el concepto de "Universalidad de Internet". Estos se traducen en que Internet debe tomar en cuenta los derechos humanos (D), ser abierta (A), ser accesible para todos (A), y alimentarse de la participación de las múltiples partes interesadas (M).

En este sentido, la dificultad a la que asistimos en la actualidad es que el posicionamiento social de las TIC favorece que los individuos que no puedan acceder a ellas puedan caer en exclusión social. La brecha digital puede convertirse en brecha social, de tal manera que la exclusión tecnológica favorecería la marginación social y personal (Cabero, 2015). Profundizando en este mismo aspecto, James (2011) analiza a nivel de país si una mayor competencia tecnológica desde una perspectiva de disminución en la brecha digital favorecería un incremento en su situación económica. Los hallazgos son reveladores: descubre que en los países donde la brecha disminuye los ingresos tienden a elevarse y viceversa. El estadístico empleado fue un análisis de regresión simple.

Existe la creencia de que la solución a los problemas está asociada con una mayor presencia de las tecnologías (Morozov, 2015). En el ámbito educativo, por ejemplo, existen indicios que señalan que una mayor disponibilidad de tecnología no se ha traducido en una transformación de prácticas educativas y la generación de nuevos escenarios de comunicación para los participantes. Además, en numerosas ocasiones los efectos de las TIC en el rendimiento de los aprendices son insustanciales o desconocidos (Barrera-Osorio y Linden, 2009; European Commission, 2008; Law et al., 2008).

Pero qué ocurre si existe una ausencia desmedida o un desajuste entre las halagüeñas estadísticas de disponibilidad de dispositivos tecnológicos y el número de esos dispositivos que contemplan la posibilidad de acceder a Internet. Es decir, un desajuste entre la tecnología presente y la tecnología que se encuentra conectada. Este aporte centra la atención en generar una breve fotografía del estado de la conectividad en el ámbito educativo del territorio español.

\section{MATERIAL Y MÉTODO}

Para abordar los objetivos se trabaja desde un planteamiento de explotación secundaria de los datos que aportan las diferentes comunidades autónomas españolas al Ministerio de Educación en el año 2017. Estos datos han sido recogidos de forma anónima, sin tomar en cuenta el nombre de las muestras del estudio. Cabe introducir que para la consecución de esta estadística estatal se estableció una cooperación activa entre el 
Ministerio de Educación y las administraciones educativas de las CC.AA. a través de la Comisión de Estadística de la Conferencia de Educación. De dicha Comisión depende el Grupo Técnico de Coordinación Estadística, formado por representantes de los servicios estadísticos del Ministerio y de las CC.AA. (MECD, 2018).

Este estudio versa sobre cómo se encuentran conectados los centros educativos desde la etapa de Educación Infantil hasta ESO y Bachillerato, midiendo diferentes variables y comparándolas entre las diferentes comunidades autónomas del país.

Para ello contamos con diferentes variables que se detallan a continuación (MECD, 2018a):

- Conexión a Internet

- Se identifica si el centro educativo tiene conexión a internet propia, o bien, se conecta por intranet a través de la comunidad autónoma.

- Según el tipo de conexión a Internet: se especificará el tipo de conexión que posee atendiendo a las siguientes opciones:

$\cdot$ RDSI

- ADSL

- Fibra óptica

- TV por cable

- Conexión de datos móvil

- Otros

- Ancho de banda: nos indica el total de ancho de banda que se dispone, tras la suma de todas las conexiones a Internet, de las siguientes:

- Menos de 2 Mbps

- Desde 2 Mbps hasta 10 Mbps

- Desde 10 Mbps hasta 20 Mbps

- Desde 20 Mbps hasta 50 Mbps

- Desde 50 Mbps hasta 100 Mbps

- Más de100 Mbps

- Conexión wifi

- Nos indica si el centro posee conexión wifi y si esta se realiza a través de Internet o de la intranet de la comunidad autónoma correspondiente.

- Lugares con acceso a internet vía wifi: se obtiene los lugares de los centros educativos que tienen conexión wifi, distinguiendo entre las siguientes opciones (se ha permitido selección múltiple)

- Aulas

- Departamentos

- Secretaría del centro y despachos de dirección

- Zonas comunes

- Personas con acceso wifi: se recogerá las personas que tienen acceso a Internet vía wifi distinguiendo entre los siguientes (se ha permitido respuesta múltiple):

- Alumnos con dispositivos del centro

- Alumnos con dispositivos propios 
- Clasificación de aulas

Se contempla como aulas cualquier zona del centro educativo donde se imparta docencia de forma habitual, teniendo en cuenta la disponibilidad de conectividad a Internet, tanto inalámbrica como cableada:

- Número de aulas con acceso a Internet

- Número de aulas sin acceso a Internet

\section{RESULTADOS}

Acorde a los objetivos planteados en el presente estudio, todos los resultados se han presentado en un único bloque, puesto que se ha considerado la mejor opción, ya que todos tratan sobre aspectos relacionados.

Lo primero que se debe recalcar es que, en referencia a las aulas con conexión a Internet, no se han tenido en cuenta las CC.AA. de Valencia y el País Vasco puesto que han aportado datos a sus correspondientes administraciones.

Sí es reseñable que Ceuta y La Rioja, con un 99,2\% y 98,9\% respectivamente, son la ciudad y comunidad autónoma con mayor conectividad. En el lado opuesto se sitúan Aragón y Canarias con unos datos de 88\% y $89 \%$ respectivamente (figura 2 ).

Cuando nos referimos al tipo de acceso a wifi, lo relevante de los datos es la escasa utilización de la intranet por parte de los centros educativos, llegando incluso a no usarse en comunidades como Andalucía y Galicia, encontrándose en su polo opuesto Melilla con un $17 \%$ de sus centros. En el lado opuesto, se muestra que la mayoría de las comunidades usan Internet en sus centros educativos, siendo Castilla la Mancha el culmen de su utilización con un $97 \%$ y la Comunidad Valenciana con un $65 \%$ la que menos. El resto de las comunidades y ciudades pueden consultarse en la figura 3.

La mayoría de las comunidades autónomas, a la hora de elegir el tipo de conexión que consideran óptimas para el desempeño de las tareas propias de los centros educativos, se decantan por la utilización de conexión ADSL, siendo la Comunidad Valenciana con un $89 \%$ su primer adalid y Cataluña como más reticente para su uso.

Figura 2. Porcentaje de aulas con conexión a Internet. Datos del MECD (2018b)

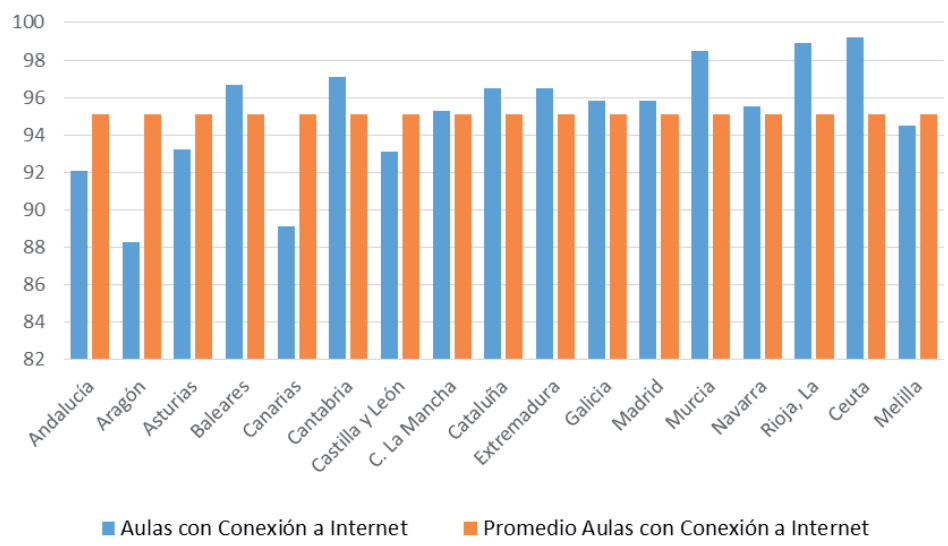

Figura 3. Porcentaje de centros según el tipo de acceso a wifi. Datos del MECD (2018b)

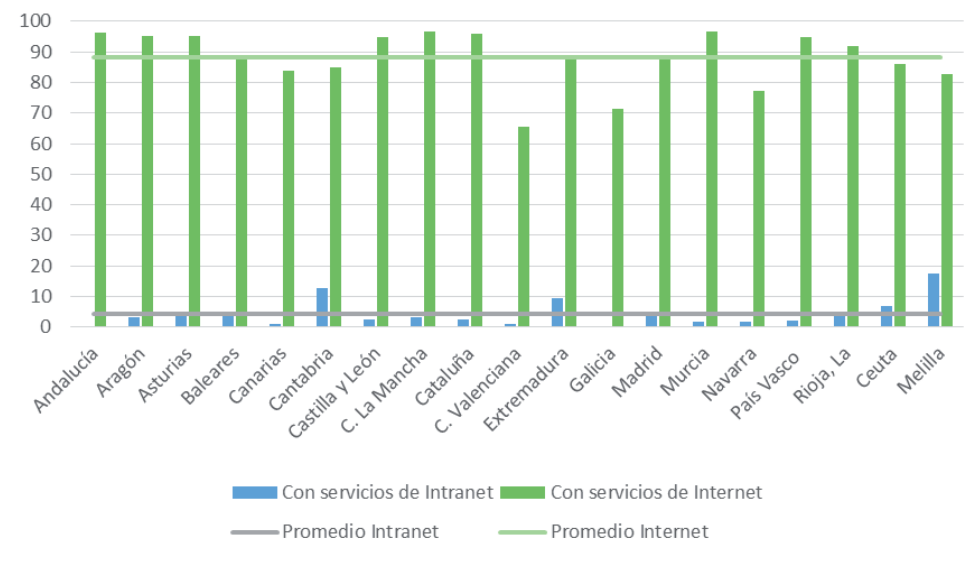


No obstante, no todas las comunidades optan por este tipo de conexión, ya que en el caso de Cataluña o Extremadura, entre otras, prefieren utilizar conexión a Internet a través de fibra óptica, suponiendo un 87 $\%$ y $72 \%$ respectivamente.

Al hablar del uso de datos móviles, se puede comprobar que es casi inexistente su utilización, salvo en el caso de Ceuta donde un 6,9\% han optado por esta opción. Un caso similar ocurre, cuando se pone en foco en el uso del RDSI, en Cataluña, Melilla o País vasco; en contraposición, Madrid con cerca del 10\% de los centros educativos que se han declinado por su utilización.

Dentro del tipo de conexiones, es reseñable Galicia, ya que en sus centros educativos un 33\% de ellos utilizan TV-Cable, mientras que en la Región de Murcia llega a 13\% la utilización de otro tipo de conexiones no especificadas (tabla 1 ).

TABLA 1. Porcentaje de centros con conexión a Internet según el tipo de conexión

\begin{tabular}{|c|c|c|c|c|c|c|}
\hline & $\begin{array}{l}\text { Conexión de } \\
\text { datos móvil }\end{array}$ & RDSI & ADSL & TV-Cable & Fibra óptica & Otra conexión \\
\hline Andalucía & 0,4 & 3,2 & 65,5 & 1,2 & 22,8 & 7,0 \\
\hline Aragón & 3,4 & 4,9 & 64,2 & 1,5 & 45,3 & 2,6 \\
\hline Asturias & 2,6 & 4,9 & 65,5 & 10,0 & 27,9 & 5,9 \\
\hline Baleares & 7,7 & 9,0 & 78,7 & 4,6 & 0,0 & 0,0 \\
\hline Canarias & 1,9 & 4,9 & 63,2 & 0,7 & 29,2 & 11,2 \\
\hline Cantabria & 2,0 & 3,6 & 51,2 & 4,8 & 57,5 & 0,8 \\
\hline Castilla y León & 4,5 & 4,1 & 80,5 & 3,3 & 24,2 & 4,0 \\
\hline C. La Mancha & 2,8 & 6,8 & 86,7 & 2,1 & 18,1 & 3,3 \\
\hline Cataluña & 4,4 & 0,0 & 22,8 & 1,1 & 87,6 & 3,0 \\
\hline C. Valenciana & 0,4 & 2,2 & 89,2 & 4,5 & 14,7 & 10,8 \\
\hline Extremadura & 2,0 & 4,1 & 33,7 & 0,5 & 72,8 & 3,1 \\
\hline Galicia & 1,3 & 1,0 & 59,1 & 33,5 & 0,7 & 4,4 \\
\hline Madrid & 5,7 & 9,8 & 47,2 & 2,8 & 64,8 & 5,3 \\
\hline Murcia & 5,0 & 3,0 & 28,0 & 3,3 & 60,9 & 13,0 \\
\hline Navarra & 1,3 & 3,4 & 63,8 & 0,0 & 33,9 & 8,1 \\
\hline País Vasco & 0,0 & 0,0 & 30,4 & 0,0 & 77,9 & 0,0 \\
\hline Rioja, La & 1,8 & 2,7 & 54,5 & 6,3 & 45,5 & 4,5 \\
\hline Ceuta & 6,9 & 3,4 & 37,9 & 0,0 & 69,0 & 0,0 \\
\hline Melilla & 0,0 & 0,0 & 43,5 & 8,7 & 56,5 & 0,0 \\
\hline
\end{tabular}

Adaptado del MECD (2018b) 
Tabla 2. Distribución porcentual de los centros según el tipo de ancho de banda

\begin{tabular}{|c|c|c|c|c|c|c|c|}
\hline & $\begin{array}{l}\text { Menos } \\
\text { de Mbps }\end{array}$ & $\begin{array}{c}\text { Desde } 2 \\
\text { Mbps hasta } \\
10 \text { Mbps }\end{array}$ & $\begin{array}{c}\text { Más de } 10 \\
\text { Mbps hasta } \\
20 \text { Mbps }\end{array}$ & $\begin{array}{l}\text { Más de } 20 \\
\text { Mbps hasta } \\
50 \text { Mbps }\end{array}$ & $\begin{array}{l}\text { Más de } 50 \text { Mbps } \\
\text { hasta } 100 \text { Mbps }\end{array}$ & $\begin{array}{l}\text { Más de } \\
100 \text { Mbps }\end{array}$ & $\begin{array}{l}\text { Descono- } \\
\text { cido }\end{array}$ \\
\hline Andalucía & 18,7 & 34,5 & 17,8 & 14,4 & 8,8 & 5,9 & 0,0 \\
\hline Aragón & 3,6 & 33,5 & 10,5 & 14,8 & 18,4 & 19,3 & 0,0 \\
\hline Asturias & 13,6 & 32,0 & 11,8 & 12,8 & 14,1 & 15,9 & 0,0 \\
\hline Baleares & 1,5 & 5,4 & 5,4 & 14,7 & 14,3 & 40,3 & 18,4 \\
\hline Canarias & 15,5 & 39,8 & 8,6 & 6,7 & 12,4 & 17,0 & 0,0 \\
\hline Cantabria & 2,8 & 18,9 & 6,8 & 16,9 & 28,1 & 26,5 & 0,0 \\
\hline Castilla y León & 5,9 & 45,5 & 12,3 & 9,5 & 10,9 & 15,9 & 0,0 \\
\hline C. La Mancha & 9,6 & 38,8 & 20,0 & 15,5 & 7,8 & 8,2 & 0,0 \\
\hline Cataluña & 2,3 & 9,5 & 8,3 & 15,4 & 36,1 & 28,3 & 0,0 \\
\hline C. Valenciana & 5,7 & 14,3 & 28,0 & 23,4 & 12,2 & 16,4 & 0,0 \\
\hline Extremadura & 2,6 & 20,5 & 9,0 & 12,1 & 34,3 & 21,5 & 0,0 \\
\hline Galicia & 2,4 & 23,8 & 22,7 & 9,1 & 0,0 & 42,0 & 0,0 \\
\hline Madrid & 2,9 & 18,7 & 10,6 & 19,3 & 23,7 & 24,8 & 0,0 \\
\hline Murcia & 0,8 & 8,7 & 10,0 & 12,7 & 30,8 & 37,0 & 0,0 \\
\hline Navarra & 0,3 & 20,8 & 9,7 & 33,9 & 25,5 & 9,7 & 0,0 \\
\hline País Vasco & 0,1 & 1,7 & 2,2 & 2,6 & 2,4 & 90,9 & 0,0 \\
\hline Rioja, La & 1,8 & 20,5 & 19,6 & 12,5 & 25,9 & 19,6 & 0,0 \\
\hline Ceuta & 3,4 & 10,3 & 6,9 & 0,0 & 17,2 & 62,1 & 0,0 \\
\hline Melilla & 0,0 & 8,7 & 0,0 & 17,4 & 17,4 & 56,5 & 0,0 \\
\hline
\end{tabular}

Tras comprobar el tipo de conexión a internet de los centros educativos, es necesario pararse para a comprar dentro de ese tipo de conexiones cual es la velocidad de esta, o ancho de banda.

En promedio, destaca como más utilizado el ancho de banda de más de 100Mbps, seguido de cerca por la conexión de entre 2-10 Mbps. No obstante, cuando uno se adentra entre las distintas opciones utilizadas por cada una de la comunidades y ciudades autónomas encuentra resultados diversos. Entre los más llamativos se encuentra que casi toda la comunidad educativa en el País Vasco una conexión de alta velocidad (91\%). En contraposición tenemos a Castilla y León, donde el porcentaje más alto de centros, con un 45\%, se sitúa en conexión de entre 2-10 Mbps. Cuando uno consulta esta serie de resultados hay un dato que destaca sobre los demás, y es que con un $18 \%$ Baleares dispone de un ancho de banda desconocido, siendo la única comunidad que dispone de este tipo de velocidad (tabla 2).

Por otro lado, cuando se analiza el tipo de dispositivo que utiliza el alumnado para conectarse a Internet, según su propiedad, da como resultado que una amplia mayoría de los alumnos utilizan los dispositivos del centro, siendo el País Vasco y Castilla la Mancha destacados en su uso, en contraposición a la Comunidad de Valencia y a Madrid. 
Innoeduca. International Journal of Technology and Educational Innovation

Soto Varela, R., Sanz Prieto, M., Boumadan Hamed, M.

Figura 4. Porcentaje de centros con acceso wifi por tipo de usuario. Datos del MECD (2018b)

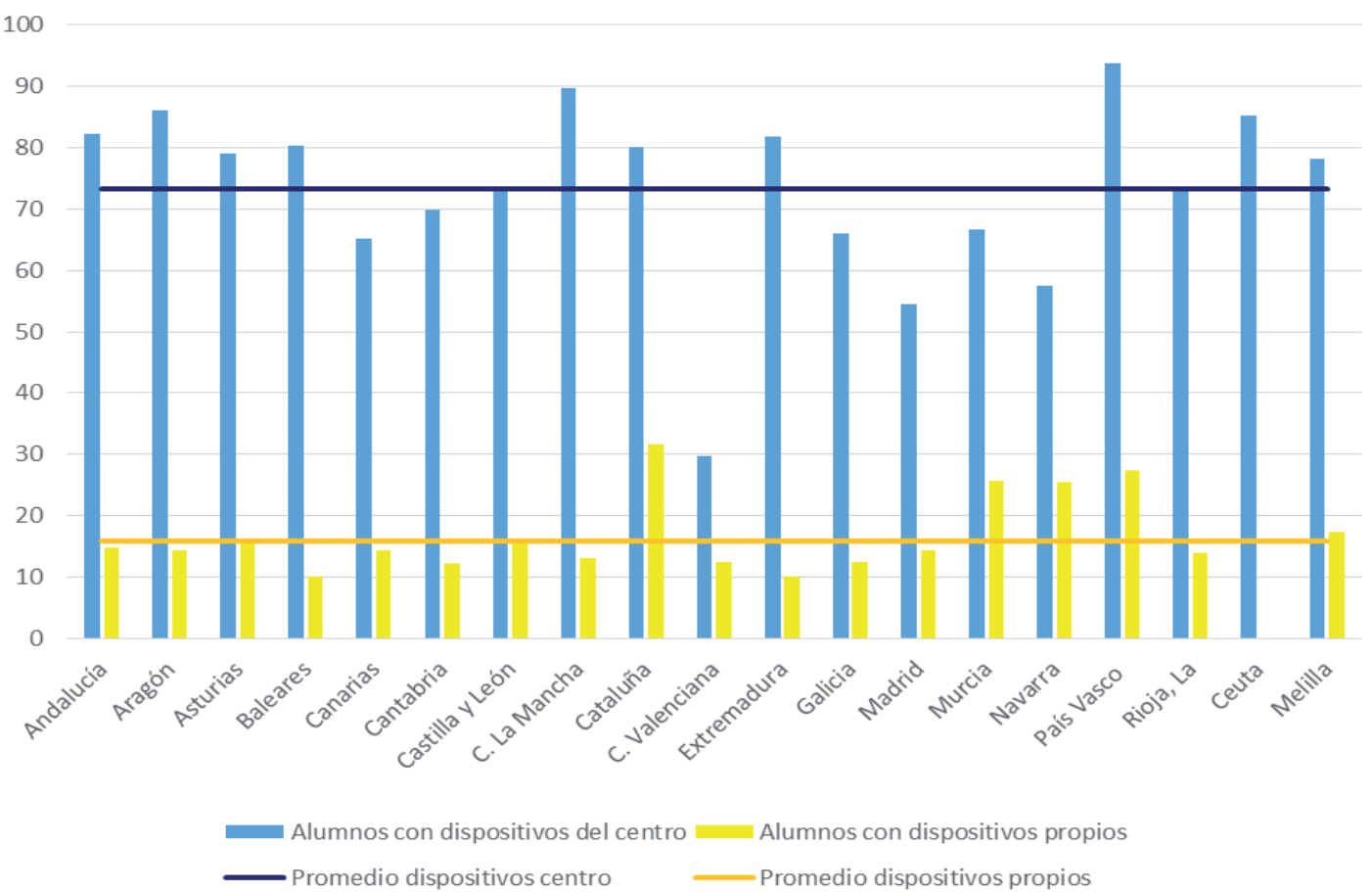

Figura 5. Porcentaje de centros con conexión wifi por zona de acceso. Datos del MECD (2018b)

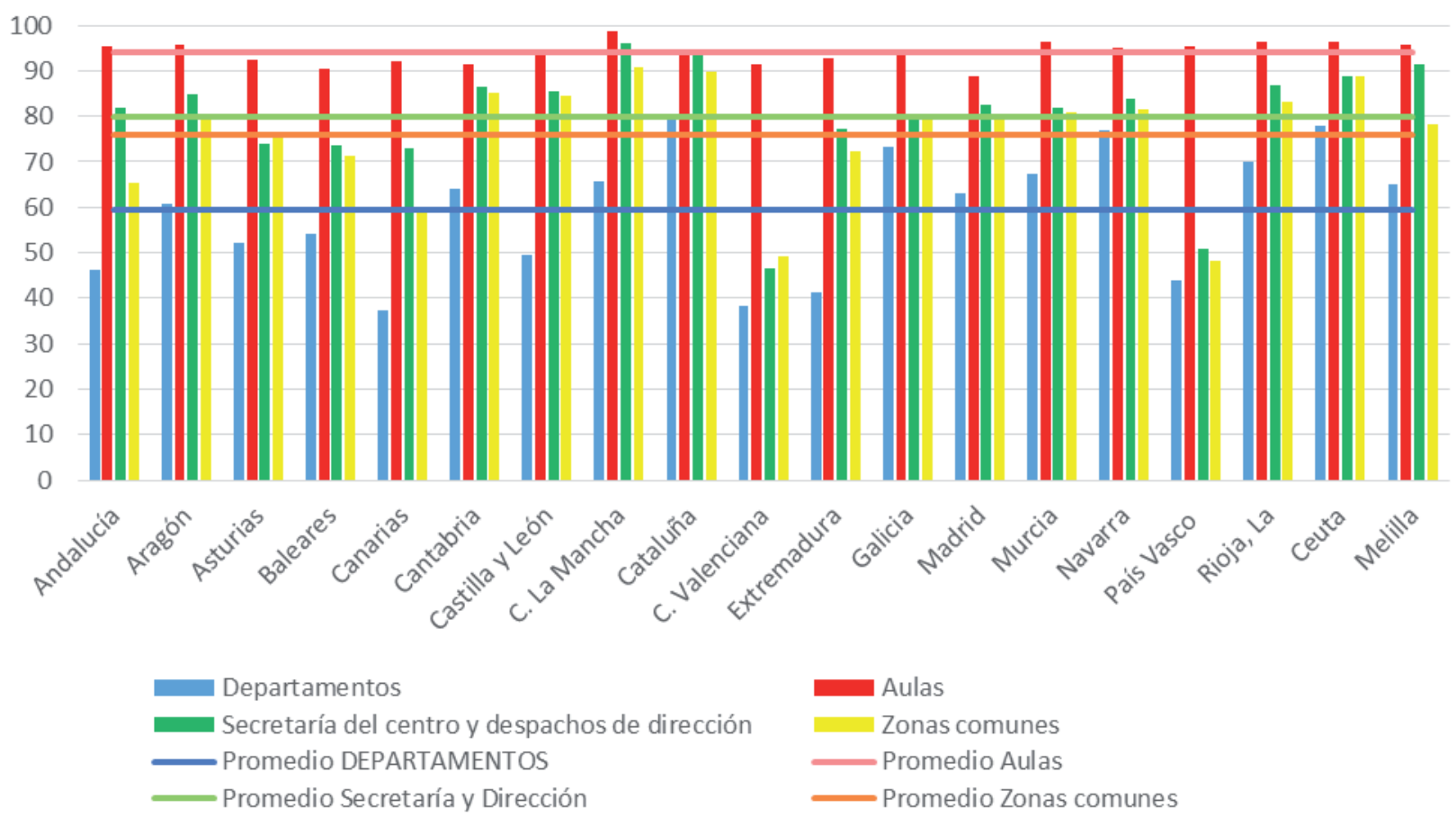


A su vez, si se observa la utilización de los propios dispositivos del alumnado, la mayoría de las comunidades se encuentra por debajo de la media; solo Cataluña, Navarra, País Vasco, Murcia y Melilla se sitúan por encima de esta; $y$, de entre ellas, es Cataluña la que se sitúa por encima del $30 \%$ de su uso. No obstante, el resultado más destacado y que más llama la atención es el hecho de que en la Ciudad Autónoma de Ceuta ningún centro educativo y, por ende, ningún alumno, utiliza sus propios dispositivos para conectarse a través de Wifi en su centro educativo de referencia (figura 4).

En cuanto a la conectividad a Internet vía wifi según la zona de acceso, la amplia mayoría de los centros hacen uso de esta tecnología dentro de las propias aulas donde se imparte docencia, con un promedio del $94 \%$. Le sigue, pero muy distanciado su uso, en las zonas destinadas para los equipos directivos donde la media se encuentra en torno al $80 \%$. Por su parte, destaca que apenas se utilice la conexión vía wifi dentro de los departamentos, donde solo un 59\% de ellos hacen este uso. Por último, en las zonas comunes y lugares de tránsito el uso del wifi se sitúa en el 76\% (figura 5).

\section{DISCUSIÓN Y CONCLUSIONES}

Hoy en día, Internet se ha convertido en una herramienta indispensable. No solo en el ámbito social, donde las redes sociales se han impuesto como los medios de información y comunicación más empleados, sino también en los entornos educativos, donde un gran número de tareas, ya sea buscar información, escuchar música, ver vídeos, utilizar juegos interactivos, leer noticias o informarse, se realizan a través de la red. Además, comunicarse o interactuar a través de archivos en la nube, ya sea entre compañeros o con la propia administración, se empieza a abordar de manera simultánea en formato "multitasking", es decir, la habilidad para hacer varias cosas a la vez.

The Survey of Schools: ICT in Education, publicado en marzo de 2019, tiene por objetivo analizar el progreso de referencia en las TIC en las escuelas en estos últimos años, el acceso, uso y las actitudes frente a la tecnología en la educación. Ponen de manifiesto la necesidad de respaldar un acceso a Internet de alta velocidad que facilite el uso de los dispositivos por parte del profesorado y de los estudiantes.

Pero no solo basta con estar conectado, la conectividad debe permitir que cualquier persona pueda realizar diferentes tareas en formato y soporte digital cómodamente (Long y Ehrmann, 2005). Para ello, esta conexión debe ser lo más rápida y estable posible, permitiendo que estas tareas puedan desarrollarse de la forma más efectiva posible.

En un estudio llevado a cabo por Escofet et al. (2019), se constata que el profesorado considera que las aulas de informática están perdiendo sentido, ya que lo adecuado es que todas las tecnologías características de este tipo de espacios estén disponibles en las aulas. A su vez, en este sentido, destacan que los docentes consideran necesario que la conexión a Internet del centro permita al alumnado acceder a la red en cualquier momento y lugar. Condición que empieza a convertirse en una realidad. Tal y como se muestra en esta investigación, por encima del 90\% de centros educativos en España hacen uso de Internet dentro de las aulas.

Estos resultados son similares a los reflejados por Barrett et al. (2017), quienes señalan que la demanda actual del profesorado se dirige hacia la necesidad de un buen nivel de calidad de la conexión a la red y la presencia de dispositivos que permitan un acceso a la información ágil y dinámica. Una situación contraria a los resultados expuestos en este trabajo, pese a las quejas del profesorado por la falta de recursos TIC y de conexión, los resultados demuestran que la gran mayoría de los centros están conectados a la red, de una u otra forma. 
En la actualidad, aún quedan bastantes centros educativos que no disponen de una conexión a Internet con una velocidad adecuada, pues una gran mayoría afirma tener una conexión inadecuada o baja (Segura et al., 2018). Argumento que concuerda con lo que reflejan los resultados de este trabajo, puesto que, a pesar de que la inmensa mayoría de los centros educativos cuentan con conexión a Internet, incluso en sus aulas, no en todas las provincias es de buena calidad, lo que implica una desigualdad dependiendo de la región. En Castilla y León casi la mitad de la población cuenta con mala conectividad. Por otro lado, el País Vasco tiene a un 90\% de la muestra estudiada conectada a una línea de Internet de alta velocidad. Esto concuerda con el trabajo desarrollado por Ruiz-Palmero et al. (2013), donde se indica que el uso de Internet se convierte en una herramienta indispensable en las aulas, no solo para buscar información, sino para el desarrollo de actividades didácticas.

A lo largo del desarrollo de este escrito se ha realizado una exhaustiva búsqueda de fuentes de información que documenten la conectividad de los centros educativos españoles, no habiendo localizado suficiente bibliografía al respecto. Podemos considerar este factor como uno de las grandes limitantes del presente trabajo.

Para finalizar, se puede concluir que la conectividad de los centros educativos españoles goza de buena salud. No obstante, cuando se avanza al detalle, hay diferencias significativas en la calidad de esta, dependiendo de la comunidad autónoma observada. Una de las grandes demandas del profesorado tiene que ver con la disponibilidad de un acceso a Internet que genere escenarios de creación e interacción fluida y ágil. Si bien parece que los datos reflejan que el acceso a Internet se está universalizando en España, no es condición suficiente para cumplir con la premisa anterior. La calidad de este acceso sigue siendo escasa en varias regiones, atentando directamente contra una agilidad y fluidez que permita establecer secuencias didácticas digitales óptimas.

En cuanto a la conectividad a Internet vía wifi según la zona de acceso, la amplia mayoría de los centros hacen uso de esta tecnología dentro de las propias aulas donde se imparte docencia, con un promedio del $94 \%$. Le sigue, pero muy distanciado, su uso en las zonas destinadas para los equipos directivos donde la media se encuentra en torno al $80 \%$. Por su parte destaca que apenas se utilice la conexión vía wifi dentro de los departamentos, donde solo un 59\% de ellos hacen este uso. Por último, las zonas comunes y lugares de tránsito por parte de toda la comunidad educativa, el uso del wifi se sitúa en el 76\%.

\section{REFERENCIAS}

Alexander, B., Ashford-Rowe, K., Barajas-Murphy, N., Dobbin, G., Knott, J., McCormack, M., Pomerantz, J., Seilhamer, R., y Weber, N. (2019). Educause Horizon Report. 2019 Higher Education Edition. Co: Educause.

Barrera-Osorio, F., y Linden, L. L. (2009). The use and misuse of computers in education: evidence from a randomized experiment in Colombia (Policy Research Working Paper, 4836). World Bank.

Barrett, P., Davies, F., Zhang, Y., y Barrett, L. (2017). The Holistic
Impact of Classroom Spaces on Learning in Specific Subjects. Environment and Behavior, 49(4), 425-451. https://doi. org/10.1177/0013916516648735

Brown, J., Ortiz-Padilla, M., y Soto-Varela, R. (2020). Does Mathematical Anxiety Differ Cross-Culturally? Journal of New Approaches in Educational ResearchK(1), 126-136. http://dx.doi. org/10.7821/naer.2020.1.464

Cabero, J. (2015). Inclusión digital - inclusión educativa. Sinergia, K, 15-18. Recuperado de http://telebachilleratoenchiapas. 
gob.mx/wpcontent/uploads/2015/08/SINERGIA2Edición.pdf.

Cabero, J., Marín, V., y Llorente, M.C. (2012). Desarrollar la competencia digital. Educación mediática a lo largo de toda la vida. Eduforma

Cabero Almenara, J., y Ruiz Palmero, J. (2017). Las Tecnologías de la Información y Comunicación para la inclusión: reformulando la brecha digital. International Journal of Educational Research and Innovation, 9, 16-30.

Escofet, A., Gros, B., López, M. y Marimon-Martí, M. (2019). Percepción del profesorado sobre la integración de la tecnología en el espacio escolar. RIITE. Revista Interuniversitaria de Investigación en Tecnología Educativa, 6, 37-47. http://dx.doi. $\operatorname{org} / 10.6018 /$ riite.360631

European Commission (2008). The education and training contribution to the Lisbon strategy. Recuperado de http:// ec.europa.eu/education/policies/2010/et_2010_en.html.

Facebook and The Economist Intelligence Unit (2019). The Inclusive Internet Index 2019. Recuperado de https://theinclusiveinternet.eiu.com

Fundación Telefónica (2019). Sociedad Digital en España 2018. Penguin Random House Grupo Editorial.

Gómez García, M., Ruiz Palmero, J., y Sánchez Rodríguez, J. (2015). Aprendizaje social en red. Las redes digitales en la formación universitaria. EDMETIC, 4(2), 71-87. https://doi. org/10.21071/edmetic.v4i2.3963

James, J. (2011). Are Changes in the Digital Divide Consistent with Global Equality or Inequality? The Information Society, 27, 121-128. doi: 10.1080/01972243.2011.548705.

Law, N., Pelgrum, W.J., y Plomo, T. (Eds.) (2008). Pedagogy and ICT in schools around the world: findings from the SITES 2006 study. CERC and Springer.

Long, P. D., y Ehrmann, S. C. (2005). Future of the learning space: Breaking out of the box. EDUCAUSE review, 40(4), 42-58.

MECD (2018a). Estadística de las Enseñanzas no universitarias. Resultados Detallados, Metodología. Curso 2016-2017. Recuperado de http://www.educacionyfp.gob.es/dam/jcr:122f2c8c26d1-409f-ad8d-170bf908f8a0/metnouni1617.pdf

MECD (2018b). Tablas y Figuras. Estadística de las Enseñanzas no universitarias. Resultados Detallados. Curso 2016-2017. Recu- perado de https://www.educacionyfp.gob.es/servicios-alciudadano/estadisticas/no-universitaria/centros/sociedadinformacion.htm

Morozov, E. (2015). La locura del solucionismo tecnológico. Clave Intelectual.

OCDE (2019). How's Life in the Digital Age? Opportunities and Risks of the Digital Transformation for People's Well-being. OECD Publishing https://doi.org/10.1787/9789264311800-en.

Orozco, G. (2015). Lo televisivo como escenario de las transformaciones entre TV y audiencias. En G. Orozco, G. (coord.), Tvmorforsis 4. Television everywhere (pp. 77-83). Productora de contenidos culturales Sagahón Repoll.

Ríos, M. (2018). Negroponte: The Man Who Would Change the World, But Everything Went Wrong. Recuperado de https:// ssrn.com/abstract $=3170428$ http://dx.doi.org/10.2139/ ssrn. 3170428

Ruiz-Palmero, J., Sánchez Rodríguez, J., y Gómez García, M. (2013). Entornos personales de aprendizaje: estado de la situación en la Facultad de Ciencias de la Educación de la Universidad de Málaga. Píxel-Bit. Revista de Medios y Educación, 42, 171-181.

Segura, M. P., Solano, I. M. y Sánchez, M. M. (2018). Uso didáctico de las TIC en los colegios rurales agrupados de la Región de Murcia. RIITE. Revista Interuniversitaria de Investigación en Tecnología Educativa, 5, 102-115. http://dx.doi.org/10.6018/ riite/2018/343771

Unesco (2019). Unesco's Internet Universality indicators. Recuperado de https://unesdoc.unesco.org/ark:/48223/ pf0000265830 